\title{
UPPER SEMISMOOTH FUNCTIONS AND THE SUBDIFFERENTIAL DETERMINATION PROPERTY
}

March 10, 2017

Marc Lassonde

Université des Antilles, BP 150, 97159 Pointe à Pitre, France; and LIMOS, Université Blaise Pascal, 63000 Clermont-Ferrand, France

E-mail: marc.lassonde@univ-ag.fr

Dedicated to the memory of Jon Borwein.

\begin{abstract}
In this paper, an upper semismooth function is defined to be a lower semicontinuous function whose radial subderivative satisfies a mild directional upper semicontinuity property. Examples of upper semismooth functions are the proper lower semicontinuous convex functions, the lower- $\mathrm{C}^{1}$ functions, the regular directionally Lipschitz functions, the Mifflin semismooth functions, the Thibault-Zagrodny directionally stable functions. It is shown that the radial subderivative of such functions can be recovered from any subdifferential of the function. It is also shown that these functions are subdifferentially determined, in the sense that if two functions have the same subdifferential and one of the functions is upper semismooth, then the two functions are equal up to an additive constant.
\end{abstract}

Keywords: upper semismooth, Dini subderivative, radial subderivative, subdifferential, subdifferential determination property, approximately convex function, regular function.

2010 Mathematics Subject Classification: 49J52, 49K27, 26D10, $26 B 25$.

\section{Introduction}

Jon Borwein discussing generalisations in the area of nonsmooth optimisation [1, p. 4] writes:

In his thesis Francis Clarke extended Moreau-Rockafellar max formula to all locally Lipschitz functions. Clarke replaced the right Dini directional derivative $D_{h} f(x)$ by

$$
D_{h}^{c} f(x)=\limsup _{0<t \rightarrow 0, y \rightarrow x} \frac{f(y+t h)-f(y)}{t} .
$$

Somewhat miraculously the mapping $p$ sending $h \rightarrow D_{h}^{c} f(x)$ is always continuous and sublinear in $h$, and so if we define $\partial^{C} f(x)=\partial p(0)=\left\{y \in X^{*}:\langle y, h\rangle \leq D_{h}^{c} f(x), \forall h \in X\right\}$, Moreau-Rockafellar max formula leads directly to:

Theorem (Clarke). Let $f: E \rightarrow \mathbb{R}$ be a locally Lipschitz function. Then

$$
D_{h}^{c} f(x)=\sup _{y \in \partial^{C} f(x)}\langle y, h\rangle
$$

for all $h \in E .[\ldots]$

In truth Clarke, appealing to Rademacher's theorem, originally defined $\partial^{C} f(x)$ as the closed convex hull of limits of nearby points of differentiability. This makes (11) seem even more remarkable. There is, however, a dark side to the situation [3, Cor. 9]. Recall that a set in a Banach space is generic if it contains intersection of countably many dense open sets. The complement is thus very small topologically. 
Theorem (Generic triviality [3]). Let $A$ be an open subset of a Banach space $X$. Then the set of non-expansive functions on $A$ with $\partial^{C} f(x) \equiv B_{X^{*}}$ for all $x$ in $A$ is generic in the uniform norm on $A$.

In other words, in the sense of Baire category the Clarke subdifferential (likewise the limiting subdifferential in the separable case) of almost all functions contains no information at any point of $A$. [...] So, for most Lipschitz functions the Clarke calculus is vacuous. That is why serious researchers work with well structured subclasses such semi-algebraic, partially smooth or essentially smooth functions.

The fact that subdifferentials cannot discriminate between functions is a serious drawback according to Terry Rockafellar [19] who argues:

In subgradient optimization, interest centers on methods for minimizing $f$ that are based on being able to generate for each $x$ at least one (but not necessarily every) $y \in \partial^{C} f(x)$, or perhaps just an approximation of such a vector $y$. One of the main hopes is that by generating a number of subgradients at various points in some neighborhood of $x$, the behavior of $f$ around $x$ can roughly be assessed. In the case of a convex function $f$ this is not just wishful thinking, and a number of algorithms, especially those of bundle type (e.g., Lemarechal 1975 and Wolfe 1975) rely on such an approach. In the nonconvex case, however, there is the possibility, without further assumptions on $f$ than local Lipschitz continuity, that the multifunction $\partial^{C} f: x \mapsto \partial^{C} f(x)$ may be rather bizarrely disassociated from $f$. An example given at the end of this section has $f$ locally Lipschitzian, yet such that there exist many other locally Lipschitzian functions $g$, not merely differing from $f$ by an additive constant, for which $\partial^{C} g(x)=\partial^{C} f(x)$ for all $x$. Subgradients alone cannot discriminate between the properties of these different functions and therefore cannot be effective in determining their local minima.

In this paper, we consider lower semicontinuous functions and arbitrary subdifferentials. We identify a large subclass of lower semicontinuous functions whose radial subderivative at a given point of their domain can be fully expressed in terms of the subdifferential of the function at neighbouring points. We show that these functions are precisely the functions whose radial subderivative satisfies a mild directional upper semicontinuity property, independently from any subdifferential. Such functions are said to be upper semismooth. This class includes the proper lower semicontinuous (directionally, approximately) convex functions, the regular directionally Lipschitz functions, the Mifflin semismooth functions, the Thibault-Zagrodny directionally stable functions.

We show that, as expected, the class of upper semismooth functions satisfies the subdifferential determination property, that is, if two functions have the same subdifferential and one of the functions is upper semismooth, then the two functions are equal up to an additive constant. J.-J. Moreau [14] was the first to consider this property for the class of proper lower semicontinuous convex functions defined on Hilbert spaces. His result was later extended by Rockafellar [16] to the same class of functions defined on general Banach spaces. Since then this property has been the object of intensive research and various classes of non convex functions have been considered in this context. We refer to the papers by L. Thibault and D. Zagrodny [21, 22] for a detailed account of the history of this property.

The technique we use to prove the subdifferential determination property is simple: from the subdifferential assumption on the two functions, one of them being upper semismooth, we derive an inequality between the radial subderivatives of the functions; we then conclude 
by invoking a mean value theorem with Dini subderivatives. The structure of the paper is as follows. In Section 2, we revisit the mean value theorems with Dini subderivatives. In Section 3. we discuss subderivatives and subdifferentials and recall the duality formula linking them. In Section 3, we define the class of upper semismooth functions and give the main examples of functions in this class. In Section 5, we prove our main theorems on the subdifferential determination property.

\section{Mean value theorems with Dini subderivatives}

The lower right-hand Dini derivative, or lower radial subderivative from the direction $d=+1$, of a function $\psi: \mathbb{R} \rightarrow]-\infty,+\infty]$ at a point $t_{0} \in \mathbb{R}$ where $\psi$ is finite is denoted by

$$
\psi^{r}\left(t_{0} ;+1\right):=\liminf _{t \searrow 0} \frac{\psi\left(t_{0}+t\right)-\psi\left(t_{0}\right)}{t} .
$$

Its upper version is denoted by

$$
\psi_{+}^{r}\left(t_{0} ;+1\right):=\limsup _{t \searrow 0} \frac{\psi\left(t_{0}+t\right)-\psi\left(t_{0}\right)}{t} .
$$

Proposition 1 (Mean value inequality). Let $\psi:[0,1] \rightarrow]-\infty,+\infty]$ be lower semicontinuous on $[0,1]$ and finite at 0 . Then, for every real number $\lambda \leq \psi(1)-\psi(0)$, there exists $t_{0} \in[0,1[$ such that $\psi\left(t_{0}\right) \leq \psi(0)+t_{0} \lambda$ and $\lambda \leq \psi^{r}\left(t_{0} ;+1\right)$.

Proof. For completeness, we recall the elementary argument, as given, e.g., in [10, Lemma 4.1] or in [11, Lemma 3.1]. For $t \in[0,1]$, let $g(t):=\psi(t)-t \lambda$. The function $g:[0,1] \rightarrow]-\infty,+\infty]$ is lsc on the compact $[0,1]$ and $g(0)=\psi(0) \leq \psi(1)-\lambda=g(1)$. Hence $g$ attains its minimum on $[0,1]$ at a point $t_{0} \neq 1$. Consequently, $\psi\left(t_{0}\right)-t_{0} \lambda=g\left(t_{0}\right) \leq g(0)=\psi(0)$ and since $g\left(t_{0}+t\right) \geq g\left(t_{0}\right)$ for every $\left.\left.t \in\right] 0,1-t_{0}\right]$, it follows that

$$
\left.\forall t \in] 0,1-t_{0}\right], \quad \frac{\psi\left(t_{0}+t\right)-\psi\left(t_{0}\right)}{t} \geq \lambda .
$$

Passing to the limit inferior as $t \searrow 0$, we get $\psi^{r}\left(t_{0} ;+1\right) \geq \lambda$ as claimed.

Proposition 2 (Mean value theorem: semicontinuous version). Let $\varphi:[0,1] \rightarrow]-\infty,+\infty]$ be lower semicontinuous on $[0,1]$ and finite at 0 and let $\gamma:[0,1] \rightarrow[-\infty,+\infty[$ be upper semicontinuous on $[0,1]$ and finite at 0 . Assume that for every $t \in[0,1[$, there exists a real number $\rho(t)$ such that $\varphi^{r}(t ;+1) \leq \rho(t) \leq \gamma^{r}(t ;+1)$. Then

$$
\varphi(1)-\varphi(0) \leq \gamma(1)-\gamma(0)
$$

Proof. Let $\psi:=\varphi-\gamma$. Then $\psi:[0,1] \rightarrow]-\infty,+\infty]$ is lower semicontinuous on $[0,1]$ and finite at 0 . So, according to Proposition [1, for every real number $r \leq \psi(1)-\psi(0)$, there exists $t_{0} \in\left[0,1\left[\right.\right.$ such that $r \leq \psi^{r}\left(t_{0} ;+1\right)$. There exists a real number $\rho\left(t_{0}\right)$ such that

$$
\varphi^{r}\left(t_{0} ;+1\right) \leq \rho\left(t_{0}\right) \leq \gamma^{r}\left(t_{0} ;+1\right)
$$


Since

$$
r \leq \psi^{r}\left(t_{0} ;+1\right)=\liminf _{t \searrow 0}\left(\frac{\varphi\left(t_{0}+t\right)-\varphi\left(t_{0}\right)}{t}-\frac{\gamma\left(t_{0}+t\right)-\gamma\left(t_{0}\right)}{t}\right)
$$

and since, by (2),

$$
\rho\left(t_{0}\right) \leq \liminf _{t \searrow 0} \frac{\gamma\left(t_{0}+t\right)-\gamma\left(t_{0}\right)}{t},
$$

for every $\varepsilon>0$ one can find $t_{\varepsilon}>0$ such that for all $\left.\left.t \in\right] 0, t_{\varepsilon}\right]$

$$
r-\varepsilon<\frac{\varphi\left(t_{0}+t\right)-\varphi\left(t_{0}\right)}{t}-\frac{\gamma\left(t_{0}+t\right)-\gamma\left(t_{0}\right)}{t} \quad \text { and } \quad \rho\left(t_{0}\right)-\varepsilon \leq \frac{\gamma\left(t_{0}+t\right)-\gamma\left(t_{0}\right)}{t},
$$

hence, for all $\left.t \in] 0, t_{\varepsilon}\right]$,

$$
r+\rho\left(t_{0}\right)-2 \varepsilon<\frac{\varphi\left(t_{0}+t\right)-\varphi\left(t_{0}\right)}{t} .
$$

Passing to the limit inferior, we get

$$
r+\rho\left(t_{0}\right)-2 \varepsilon \leq \varphi^{r}\left(t_{0} ;+1\right) .
$$

Since $\varphi^{r}\left(t_{0} ;+1\right) \leq \rho\left(t_{0}\right)$ by (2), it follows that $r-2 \varepsilon \leq 0$. As $\varepsilon>0$ was arbitrary, we conclude that $r \leq 0$. Therefore, for every real number $r \leq \psi(1)-\psi(0)$ one has $r \leq 0$, proving that $\psi(1)-\psi(0) \leq 0$. This amounts to $\varphi(1)-\varphi(0) \leq \gamma(1)-\gamma(0)$, as claimed.

Remark 2.1. (a) In Proposition 2, the semicontinuity conditions on $\varphi$ and $\gamma$ cannot be relaxed. Indeed, consider the functions $\varphi, \gamma:[0,1] \rightarrow \mathbb{R}$ defined by

$$
\varphi(t):=0 \quad \text { for all } t \in[0,1] \text { and } \gamma(t):=\left\{\begin{array}{ll}
1 & \text { if } t \in[0,1 / 2[ \\
0 & \text { if } t \in[1 / 2,1]
\end{array} .\right.
$$

Then, $\varphi:[0,1] \rightarrow \mathbb{R}$ is continuous, $\gamma:[0,1] \rightarrow \mathbb{R}$ is continuous except at point $1 / 2$ where it is merely right-continuous, and $\varphi^{r}(t ;+1)=\gamma^{r}(t ;+1)=0$ for all $t \in[0,1[$. Yet the conclusion in Proposition 2 is false: $\varphi(1)-\varphi(0)=0>\gamma(1)-\gamma(0)=-1$.

(b) In Proposition 2, the finiteness conditions $\varphi^{r}(t ;+1)<+\infty$ and $\gamma^{r}(t ;+1)>-\infty$ for every $t \in[0,1[$, cannot be relaxed. Indeed, consider the functions $\varphi, \gamma:[0,1] \rightarrow \mathbb{R}$ defined by

$$
\varphi(t):=\left\{\begin{array}{ll}
2 & \text { if } t \in] 0,1] \\
0 & \text { if } t=0
\end{array} \quad \text { and } \quad \gamma(t)=\sqrt{t} \quad \text { for all } t \in[0,1]\right.
$$

Then, $\varphi:[0,1] \rightarrow]-\infty,+\infty]$ is lower semicontinuous on $[0,1]$ with finite values, $\gamma:[0,1] \rightarrow \mathbb{R}$ is continuous and $\varphi^{r}(t ;+1) \leq \gamma^{r}(t ;+1)$ for every $t \in\left[0,1\right.$. Moreover, $\varphi^{r}(t ;+1)$ and $\gamma^{r}(t ;+1)$ are finite for every $t \in] 0,1\left[\right.$, but $\varphi^{r}(0 ;+1)=\gamma^{r}(0 ;+1)=+\infty$, and indeed the conclusion of Proposition 2 is false: $\varphi(1)-\varphi(0)=2>\gamma(1)-\gamma(0)=1$.

Proposition 3 (Mean value theorem: continuous version). Let $\varphi, \gamma:[0,1] \rightarrow \mathbb{R}$ be continuous. Assume there is a countable subset $C \subset[0,1]$ such that for every $t \in[0,1] \backslash C$, there exists a real number $\rho(t)$ such that $\varphi_{+}^{r}(t ;+1) \leq \rho(t) \leq \gamma^{r}(t ;+1)$. Then

$$
\varphi(1)-\varphi(0) \leq \gamma(1)-\gamma(0) \text {. }
$$


Proof. We follow the pattern of the proof of [8, (8.5.1)]. For any $\varepsilon>0$, we will show that

$$
\varphi(1)-\varphi(0) \leq \gamma(1)-\gamma(0)+3 \varepsilon
$$

the left hand side being independent of $\varepsilon$, this will complete the proof. Let $C:=\left\{c_{n}: n \in \mathbb{N}\right\}$ be the given countable subset of $[0,1]$. Consider the set

$$
A:=\left\{t \in[0,1]: \forall t^{\prime} \in[0, t], \varphi\left(t^{\prime}\right)-\varphi(0) \leq \gamma\left(t^{\prime}\right)-\gamma(0)+\varepsilon t^{\prime}+\varepsilon \sum_{c_{n}<t^{\prime}} 2^{-n}\right\} .
$$

It is clear that $0 \in A$ and that if $t \in A$, then $[0, t] \subset A$. Let $t_{0}=\sup A$. From the continuity of $\varphi$ and $\gamma$ it follows that $t_{0} \in A$, so $\left[0, t_{0}\right]=A$. Therefore we need only prove that $t_{0}=1$.

Suppose $t_{0}<1$. If $t_{0} \notin C$, there is a real number $\rho\left(t_{0}\right)$ such that

$$
\varphi_{+}^{r}\left(t_{0} ;+1\right) \leq \rho\left(t_{0}\right) \leq \gamma^{r}\left(t_{0} ;+1\right),
$$

so, by definition of the subderivatives, we can find $\eta>0$ such that for every $s \in] 0, \eta]$,

$$
\varphi\left(t_{0}+s\right)-\varphi\left(t_{0}\right) \leq\left(\rho\left(t_{0}\right)+\varepsilon / 2\right) s \quad \text { and } \quad\left(\rho\left(t_{0}\right)-\varepsilon / 2\right) s \leq \gamma\left(t_{0}+s\right)-\gamma\left(t_{0}\right),
$$

hence

$$
\varphi\left(t_{0}+s\right)-\varphi\left(t_{0}\right) \leq \gamma\left(t_{0}+s\right)-\gamma\left(t_{0}\right)+\varepsilon s,
$$

and since $t_{0} \in A$, we deduce

$$
\begin{aligned}
\varphi\left(t_{0}+s\right)-\varphi(0) & \leq \gamma\left(t_{0}+s\right)-\gamma(0)+\varepsilon\left(t_{0}+s\right)+\varepsilon \sum_{c_{n}<t_{0}} 2^{-n} \\
& \leq \gamma\left(t_{0}+s\right)-\gamma(0)+\varepsilon\left(t_{0}+s\right)+\varepsilon \sum_{c_{n}<t_{0}+s} 2^{-n},
\end{aligned}
$$

hence $t_{0}+\eta \in A$ contrary to the definition of $t_{0}$. If $t_{0} \in C$, the set $\left\{n \in \mathbb{N}: t_{0}=c_{n}\right\}$ is not empty; by continuity of $\varphi$ and $\gamma$, we can find $\eta>0$ such that for every $s \in] 0, \eta]$,

$$
\varphi\left(t_{0}+s\right)-\varphi\left(t_{0}\right) \leq(\varepsilon / 2) \sum_{c_{n}=t_{0}} 2^{-n} \quad \text { and } \quad 0 \leq \gamma\left(t_{0}+s\right)-\gamma\left(t_{0}\right)+(\varepsilon / 2) \sum_{c_{n}=t_{0}} 2^{-n},
$$

hence from $t_{0} \in A$ we deduce again

$$
\begin{aligned}
\varphi\left(t_{0}+s\right)-\varphi(0) & \leq \gamma\left(t_{0}+s\right)-\gamma(0)+\varepsilon t_{0}+\varepsilon \sum_{c_{n}<t_{0}+s} 2^{-n} \\
& \leq \gamma\left(t_{0}+s\right)-\gamma(0)+\varepsilon\left(t_{0}+s\right)+\varepsilon \sum_{c_{n}<t_{0}+s} 2^{-n},
\end{aligned}
$$

which is a contradiction. 


\section{Subderivatives and subdifferentials}

In the sequel, $X$ is a real Banach space, $X^{*}$ is its topological dual, and $\langle.,$.$\rangle is the duality$ pairing. For $x, y \in X$, we let $[x, y]:=\{x+t(y-x): t \in[0,1]\}$; the sets $] x, y[$ and $[x, y[$ are defined accordingly. Set-valued operators $T: X \rightrightarrows X^{*}$ are identified with their graph $T \subset X \times X^{*}$. All extended-real-valued functions $\left.\left.f: X \rightarrow\right]-\infty,+\infty\right]$ are assumed to be lower semicontinuous (lsc) and proper, which means that the set $\operatorname{dom} f:=\{x \in X: f(x)<\infty\}$ is non-empty.

The framework, terminology and notation are the same as in our work [12]. For the reader's convenience, we briefly recall the main definitions and facts.

For a lsc function $f: X \rightarrow]-\infty,+\infty]$, a point $\bar{x} \in \operatorname{dom} f$ and a direction $u \in X$, we consider the following basic subderivatives:

- the (lower right Dini) radial subderivative:

$$
f^{r}(\bar{x} ; u):=\liminf _{t \searrow 0} \frac{f(\bar{x}+t u)-f(\bar{x})}{t},
$$

its upper version:

$$
f_{+}^{r}(\bar{x} ; u):=\limsup _{t \searrow 0} \frac{f(\bar{x}+t u)-f(\bar{x})}{t},
$$

and its upper strict version (the Clarke subderivative):

$$
f^{0}(\bar{x} ; u):=\limsup _{\substack{t \searrow 0 \\(x, f(x)) \rightarrow(\bar{x}, f(\bar{x}))}} \frac{f(x+t u)-f(x)}{t} ;
$$

- the (lower right Dini-Hadamard) directional subderivative:

$$
f^{d}(\bar{x} ; u):=\liminf _{\substack{t \searrow 0 \\ u^{\prime} \rightarrow u}} \frac{f\left(\bar{x}+t u^{\prime}\right)-f(\bar{x})}{t},
$$

and its upper strict version (the Clarke-Rockafellar subderivative):

$$
f^{\uparrow}(\bar{x} ; u):=\sup _{\delta>0} \limsup _{\substack{t \searrow 0 \\(x, f(x)) \rightarrow(\bar{x}, f(\bar{x}))}} \inf _{u^{\prime} \in B_{\delta}(u)} \frac{f\left(x+t u^{\prime}\right)-f(x)}{t} .
$$

It is immediate from these definitions that the following inequalities hold $(\rightarrow$ means $\leq)$ :

$$
\begin{aligned}
& f^{r}(\bar{x} ; u) \rightarrow f_{+}^{r}(\bar{x} ; u) \rightarrow f^{0}(\bar{x} ; u) \\
& \uparrow \quad \uparrow \\
& f^{d}(\bar{x} ; u) \quad \longrightarrow \quad f^{\uparrow}(\bar{x} ; u)
\end{aligned}
$$

For $f$ locally Lipschitz at $\bar{x}$, one has $f^{r}(\bar{x} ; u)=f^{d}(\bar{x} ; u)$ and $f^{0}(\bar{x} ; u)=f^{\uparrow}(\bar{x} ; u)$. For $f$ lsc convex, one has $f^{d}(\bar{x} ; u)=f^{\uparrow}(\bar{x} ; u)$. A function $f$ satisfying such an equality is called regular. However, in general, $f^{d}(\bar{x} ; u)<f^{\uparrow}(\bar{x} ; u)$. 
Next, given a lsc function $f: X \rightarrow]-\infty,+\infty]$ and a point $\bar{x} \in \operatorname{dom} f$, we consider the following two basic subsets of the dual space $X^{*}$ :

- the Moreau-Rockafellar subdifferential (the subdifferential of convex analysis):

$$
\partial_{M R} f(\bar{x}):=\left\{x^{*} \in X^{*}:\left\langle x^{*}, y-\bar{x}\right\rangle+f(\bar{x}) \leq f(y), \forall y \in X\right\}
$$

- the Clarke subdifferential, associated to the Clarke-Rockafellar subderivative:

$$
\partial_{C} f(\bar{x}):=\left\{x^{*} \in X^{*}:\left\langle x^{*}, u\right\rangle \leq f^{\uparrow}(\bar{x} ; u), \forall u \in X\right\} .
$$

All the classical subdifferentials (proximal, Fréchet, Hadamard, Ioffe, Michel-Penot, ... ) lie between these two subsets, and for a lsc convex $f$, all these subdifferentials coincide.

In the sequel, we call subdifferential any operator $\partial$ that associates a set-valued mapping $\partial f: X \rightrightarrows X^{*}$ to each function $f$ on $X$ so that

$$
\partial_{M R} f \subset \partial f \subset \partial_{C} f
$$

and the following Separation Principle is satisfied on $X$ :

(SP) For any lsc $f, \varphi$ with $\varphi$ convex Lipschitz near $\bar{x} \in \operatorname{dom} f$, if $f+\varphi$ admits a local minimum at $\bar{x}$, then $0 \in \widehat{\partial} f(\bar{x})+\partial \varphi(\bar{x})$, where

$$
\begin{aligned}
& \widehat{\partial} f(\bar{x}):=\left\{\bar{x}^{*} \in X^{*} \text { : there is a net }\left(\left(x_{\nu}, x_{\nu}^{*}\right)\right)_{\nu} \subset \partial f\right. \text { with } \\
& \left.\qquad\left(x_{\nu}, f\left(x_{\nu}\right)\right) \rightarrow(\bar{x}, f(\bar{x})), x_{\nu}^{*} \stackrel{w^{*}}{\longrightarrow} \bar{x}^{*}, \limsup _{\nu}\left\langle x_{\nu}^{*}, x_{\nu}-\bar{x}\right\rangle \leq 0\right\} .
\end{aligned}
$$

The Clarke subdifferential, the Michel-Penot (moderate) subdifferential and the Ioffe subdifferential satisfy the Separation Principle in any Banach space. The elementary subdifferentials (proximal, Fréchet, Hadamard, ...), as well as their viscosity and limiting versions, satisfy the Separation Principle in appropriate Banach spaces. See, e.g. [9, 10, 15] and the references therein.

Subdifferentials satisfying the Separation Principle are densely defined:

Theorem 4 (Density of subdifferentials). Let $X$ be a Banach space, $f: X \rightarrow]-\infty,+\infty]$ be lsc and $\bar{x} \in \operatorname{dom} f$. Then, there exists a sequence $\left(\left(x_{n}, x_{n}^{*}\right)\right)_{n} \subset \partial f$ such that $x_{n} \rightarrow \bar{x}$, $f\left(x_{n}\right) \rightarrow f(\bar{x})$ and $\lim \sup _{n}\left\langle x_{n}^{*}, x_{n}-\bar{x}\right\rangle \leq 0$.

Proof. See [11, Theorem 2.1].

A sequence $\left(x_{n}\right) \subset X$ is said to be directionally convergent to $\bar{x}$ in the direction $v \in X$, written $x_{n} \rightarrow_{v} \bar{x}$, if there are two sequences $t_{n} \searrow 0$ (that is, $t_{n} \rightarrow 0$ with $t_{n}>0$ ) and $v_{n} \rightarrow v$ such that $x_{n}=\bar{x}+t_{n} v_{n}$ for all $n$. Observe that for $v=0, x_{n} \rightarrow_{v} \bar{x}$ simply means $x_{n} \rightarrow \bar{x}$.

We call subderivative associated to a subdifferential $\partial f$ at a point $(\bar{x}, u) \in \operatorname{dom} f \times X$, the support function of the set $\partial f(\bar{x})$ in the direction $u$, which we denote by

$$
f^{\partial}(\bar{x} ; u):=\sup \left\{\left\langle\bar{x}^{*}, u\right\rangle: \bar{x}^{*} \in \partial f(\bar{x})\right\} .
$$

Subderivatives and subdifferentials are linked by the following formula where, given $f$ : $X \rightarrow]-\infty,+\infty]$, we have denoted by $f^{\prime}: \operatorname{dom} f \times X \rightarrow \overline{\mathbb{R}}$ any function lying between the subderivatives $f^{d}$ and $f^{\uparrow}$, that is: $f^{d} \leq f^{\prime} \leq f^{\uparrow}$ : 
Theorem 5 (Subderivative-subdifferential duality formula). Let $X$ be a Banach space, $f$ : $X \rightarrow]-\infty,+\infty]$ be lsc, $\bar{x} \in \operatorname{dom} f$ and $u \in X$. Then, for any direction $v \in X$ and any real number $\alpha \geq 0$, one has

$$
\begin{aligned}
\limsup _{x \rightarrow v \bar{x}} f^{r}(x ; u+\alpha(\bar{x}-x)) & =\limsup _{\substack{x \rightarrow v \bar{x} \\
f^{\prime}}}(x ; u+\alpha(\bar{x}-x)) \\
& =\limsup _{x \rightarrow v \bar{x}} f^{\partial}(x ; u+\alpha(\bar{x}-x)) .
\end{aligned}
$$

Proof. See [12, Theorem 3].

Remark 5.1. (a) For $f$ locally Lipschitz at $\bar{x}$, the formulas (3) do not depend on $\alpha \geq 0$ since

$$
\limsup _{x \rightarrow v \bar{x}} f^{r}(x ; u+\alpha(\bar{x}-x))=\limsup _{x \rightarrow v \bar{x}} f^{r}(x ; u),
$$

but they may depend on the direction $v \in X$ : for $f: x \in \mathbb{R} \mapsto f(x):=-|x|$ and $u \neq 0$, one has

$$
\limsup _{x \rightarrow u 0} f^{r}(x ; u)=-|u|<\limsup _{x \rightarrow 0} f^{r}(x ; u)=|u| .
$$

(b) For arbitrary lsc $f$, the value of the expressions in (3) may depend on $\alpha \geq 0$ even for convex $f$. Indeed, in general for a lsc convex $f$ one has

$$
f^{r}(\bar{x} ; u)<\limsup _{x \rightarrow \bar{x}} f^{r}(x ; u),
$$

while always (see [12, Proposition 4])

$$
f^{r}(\bar{x} ; u)=\inf _{\alpha \geq 0} \limsup _{x \rightarrow \bar{x}} f^{r}(x ; u+\alpha(\bar{x}-x)) .
$$

\section{Upper semismooth functions}

A lsc function $f: X \rightarrow]-\infty,+\infty]$ is said to be radially accessible at a point $\bar{x} \in \operatorname{dom} f$ from a direction $u \in X$ provided

$$
f(\bar{x})=\liminf _{t \searrow 0} f(\bar{x}+t u)
$$

or equivalently, provided there exists a sequence $t_{n} \searrow 0$ such that $f\left(\bar{x}+t_{n} u\right) \rightarrow f(\bar{x})$. Every convex lsc function $f: X \rightarrow]-\infty,+\infty]$ is radially accessible at $\bar{x}$ from any $u \in X$ such that $\bar{x}+u \in \operatorname{dom} f$. On the other hand, it is easily seen that if a lsc $f$ satisfies $f^{r}(\bar{x} ; u)<+\infty$, then $f$ is radially accessible at $\bar{x}$ from $u$. The converse is not true: the function $f: t \in \mathbb{R} \mapsto \sqrt{|t|}$ is continuous, yet $f^{r}(0 ; u)=+\infty$. For more examples and properties, see [12].

Proposition 6 (Radial stability of the upper radial subderivative). Let $X$ be a Hausdorff locally convex space, $f: X \rightarrow]-\infty,+\infty]$ be lsc, $\bar{x} \in \operatorname{dom} f$ and $u \in X$ such that $f$ is radially accessible at $\bar{x}$ from $u$. Then, there is a sequence $\mu_{n} \searrow 0$ such that $f\left(\bar{x}+\mu_{n} u\right) \rightarrow f(\bar{x})$ and

$$
f_{+}^{r}(\bar{x} ; u) \leq \liminf _{n \rightarrow+\infty} f^{r}\left(\bar{x}+\mu_{n} u ; u\right) .
$$

In particular,

$$
f_{+}^{r}(\bar{x} ; u) \leq \inf _{\alpha \geq 0} \limsup _{x \rightarrow u} f^{r}(x ; u+\alpha(\bar{x}-x)) .
$$


Proof. See [12, Proposition 7].

Theorem 5. Formula (4) for convex lsc functions and Proposition 6 suggest to consider the following class of lsc functions.

Definition 1. A lsc function $f: X \rightarrow]-\infty,+\infty]$ is said to be upper semismooth at a point $\bar{x} \in \operatorname{dom} f$ in the direction $u \in X$ provided

$$
\inf _{\alpha \geq 0} \limsup _{x \rightarrow u} f^{r}(x ; u+\alpha(\bar{x}-x)) \leq f^{r}(\bar{x} ; u) .
$$

Remark 6.1. (a) In case $f$ is locally Lipschitz at $\bar{x}$, (7) boils down to

$$
\limsup _{x \rightarrow u \bar{x}} f^{r}(x ; u) \leq f^{r}(\bar{x} ; u) .
$$

We then essentially recover the class of locally Lipschitz upper semismooth functions considered by Borwein-Moors [2, p. 305] (with the slight difference that the upper radial subderivative $f_{+}^{r}$ is used there instead of the lower radial subderivative $f^{r}$ used here). As observed in [2, p. 305], the terminology is justified by the characterization of semismooth functions given by Correa-Jofré [5, Corollary 6.3] (see also Proposition 7(d) below).

(b) In case $f$ is radially accessible at $\bar{x}$ from the direction $u$, the inequality (7) becomes:

$$
f^{r}(\bar{x} ; u)=\inf _{\alpha \geq 0} \limsup _{x \rightarrow u \bar{x}} f^{r}(x ; u+\alpha(\bar{x}-x)),
$$

and in addition, $f^{r}(\bar{x} ; u)=f_{+}^{r}(\bar{x} ; u)$, i.e. the lower radial derivative and its upper version coincide at $\bar{x}$ in the direction $u$. This follows by combining (17) with the inequality (6) in Proposition 6 .

(c) One can have $f^{r}(\bar{x} ; u)= \pm \infty$ in (7). For example, the continuous function $x \mapsto \sqrt{|x|}$ is upper semismooth at $\bar{x}=0$ in the direction $u=1$ with $f^{r}(0 ; 1)=+\infty$, while the continuous function $x \mapsto-\sqrt{|x|}$ is upper semismooth at $\bar{x}=0$ in the direction $u=1$ with $f^{r}(0 ; 1)=-\infty$.

We shall also consider a strict variant of the above notion:

Definition 2. A lsc function $f: X \rightarrow]-\infty,+\infty$ ] is said to be strictly upper semismooth at a point $\bar{x} \in \operatorname{dom} f$ in the direction $u \in X$ provided

$$
\inf _{\alpha \geq 0} \limsup _{x \rightarrow \bar{x}} f^{r}(x ; u+\alpha(\bar{x}-x)) \leq f^{r}(\bar{x} ; u) .
$$

In fact, equality holds in (8) since the reverse inequality is always true.

Remark 6.2. (a) As above, in case $f$ is locally Lipschitz at $\bar{x}$, (8) boils down to

$$
\limsup _{x \rightarrow \bar{x}} f^{r}(x ; u) \leq f^{r}(\bar{x} ; u) .
$$

Since $\lim \sup _{x \rightarrow \bar{x}} f^{r}(x ; u)=f^{0}(\bar{x} ; u)$ according to Borwein-Strójwas [4, Theorem 2.1], the inequality (8Lip) is actually equivalent to the equality $f^{r}(\bar{x} ; u)=f^{0}(\bar{x} ; u)$. The terminology is therefore justified since the latter equality means that the lower radial subderivative and its upper strict version coincide at $\bar{x}$ in the direction $u$. Locally Lipschitz functions satisfying such an equality in every direction $u \in X$ are called (Clarke) regular at $\bar{x}$ (see also below the extension of this concept to the general case of lsc functions). 
(b) Evidently, (8) is more demanding than (7), so every strictly upper semismooth function is upper semismooth. The converse is not true: the locally Lipschitz function $f: x \in \mathbb{R} \mapsto$ $-|x|$ satisfies

$$
\limsup _{x \rightarrow u 0} f^{r}(x ; u)=-|u|=f^{r}(0 ; u)<\limsup _{x \rightarrow 0} f^{r}(x ; u)=|u|,
$$

so $f$ is upper semismooth at $\bar{x}=0$ from any $u \neq 0$ but not strictly upper semismooth.

We now proceed to give examples of strictly and non-strictly upper semismooth functions. Let us recall the definition of the concepts we shall consider. Let $X$ be a Banach space, $f: X \rightarrow]-\infty,+\infty]$ be lsc, $\bar{x} \in \operatorname{dom} f$ and $u \in X, u \neq 0$. Then $f$ is said to be:

- semismooth (Mifflin [13]) at $\bar{x}$ provided $f$ is locally Lipschitz and for all $u \in X$,

$$
\left(\left(x_{n}, x_{n}^{*}\right)\right) \subset \partial_{C} f \text { with } x_{n} \rightarrow_{u} \bar{x} \Rightarrow\left\langle x_{n}^{*}, u\right\rangle \rightarrow f^{r}(\bar{x} ; u) .
$$

- directionally Lipschitz at $\bar{x}$ with respect to $u$ (Rockafellar [18]) if $f$ is lsc and

$$
\limsup _{\substack{(x, f(x)) \rightarrow(\bar{x}, f(\bar{x})) \\ t \searrow 0, v \rightarrow u}} \frac{f(x+t v)-f(x)}{t}<\infty .
$$

- regular at $\bar{x}$ (Rockafellar [17]) if $f^{d}(\bar{x} ; v)=f^{\uparrow}(\bar{x} ; v)$ for every $v \in X$.

- directionally approximately convex at $\bar{x}$ (see Daniilidis-Georgiev [6], Daniilidis-JulesLassonde [7] and the references therein), if for every $u \in S_{X}$ and $\varepsilon>0$ there exists $\delta>0$ such that for all $x, y \in B(\bar{x}, \delta)$, with $x \neq y$ and $(x-y) /\|x-y\| \in B(u, \delta)$, and all $t \in[0,1]$

$$
f(t x+(1-t) y) \leq t f(x)+(1-t) f(y)+\varepsilon t(1-t)\|x-y\| .
$$

(In finite-dimensional spaces, a locally Lipschitz function is (directionally) approximately convex if and only if it is lower- $C^{1}$, cf. [20, 6].)

Proposition 7 (Examples of (strictly) upper semismooth functions). Let $X$ be a Banach space, $f: X \rightarrow]-\infty,+\infty]$ be $l s c, \bar{x} \in \operatorname{dom} f$ and $u \in X$ with $u \neq 0$ and $\bar{x}+u \in \operatorname{dom} f$. Each of the following $f$ is strictly upper semismooth at $\bar{x}$ in the direction $u$ :

(a) $f$ is directionally Lipschitz at $\bar{x}$ with respect to $u$ and regular at $\bar{x}$;

(b) $f$ is convex;

(c) $f$ is directionally approximately convex.

The following $f$ is upper semismooth at $\bar{x}$ in the direction $u$ :

(d) $f$ is locally Lipschitz and semismooth at $\bar{x}$.

Proof. (a) For $f$ directionally Lipschitz at $\bar{x}$ with respect to $u$, one has $f^{\uparrow}(\bar{x} ; u)=f^{0}(\bar{x} ; u)$ (cf. [18, Theorem 3]). If moreover $f$ is regular at $\bar{x}$, that is $f^{d}(\bar{x} ; v)=f^{\uparrow}(\bar{x} ; v)$ for every $v \in X$, we derive that $f^{d}(\bar{x} ; u)=f^{0}(\bar{x} ; u)$. Then,

$$
f^{r}(\bar{x} ; u) \geq f^{d}(\bar{x} ; u)=f^{0}(\bar{x} ; u)=\limsup _{x \rightarrow \bar{x}} f^{r}(x ; u) \geq \inf _{\alpha \geq 0} \limsup _{x \rightarrow \bar{x}} f^{r}(x ; u+\alpha(\bar{x}-x)) .
$$

Hence (8) is satisfied. 
(b) We know from [12, Proposition 4] that, if $f$ is lsc convex, then

$$
f^{r}(\bar{x} ; u)=\inf _{\alpha \geq 0} \limsup _{x \rightarrow \bar{x}} f^{r}(x ; u+\alpha(\bar{x}-x)) .
$$

Hence, (8) holds.

(c) A directionally approximately convex function satisfies the following property (cf. [7, Proposition 1]): for every $\epsilon>0$ and $u \in S_{X}$ there exists $\delta>0$ such that for all $x \in B(\bar{x}, \delta)$ and all $v \neq 0$ so that $x+v \in B(\bar{x}, \delta)$ and $v /\|v\| \in B(u, \delta)$, one has

$$
f^{\uparrow}(x ; v) \leq f(x+v)-f(x)+\varepsilon\|v\| .
$$

Without loss of generality, we may assume that the given $u$ belongs to $S_{X}$. Let $\epsilon>0$ and $\delta>0$ such that (11) holds. Fix $0<t<\delta$ and consider any $x \in B(\bar{x}, t \delta / 2)$. Let $v=t u+\bar{x}-x$. Then $x+v \in B(\bar{x}, \delta)$ and $v=t(u+(\bar{x}-x) / t \in] 0, \delta B(u, \delta)$ [. It follows from (11) that for any such $x$,

$$
f^{\uparrow}(x ; t u+\bar{x}-x) \leq f(\bar{x}+t u)-f(x)+\varepsilon\|t u+\bar{x}-x\| .
$$

Hence, for every $0<t<\delta$, since $f$ is lsc at $\bar{x}$,

$$
\limsup _{x \rightarrow \bar{x}} f^{\uparrow}(x ; t u+\bar{x}-x) \leq f(\bar{x}+t u)-f(\bar{x})+t \varepsilon .
$$

So, for every $\varepsilon>0$,

$$
\liminf _{t \searrow 0} \limsup _{x \rightarrow \bar{x}} f^{\uparrow}\left(x ; \frac{t u+\bar{x}-x}{t}\right) \leq \liminf _{t \searrow 0} \frac{f(\bar{x}+t u)-f(\bar{x})}{t}+\varepsilon .
$$

Consequently,

$$
\inf _{\alpha \geq 0} \limsup _{x \rightarrow \bar{x}} f^{\uparrow}(x ; u+\alpha(\bar{x}-x)) \leq f^{r}(\bar{x} ; u) .
$$

Hence (8) holds since we can replace $f^{\uparrow}$ by $f^{r}$ in the left hand side according to Theorem 5 ,

(d) By [5, Corollary 6.3], a locally Lipschitz function $f$ is semismooth if and only if

$$
f^{r}(\bar{x} ; u)=\lim _{x \rightarrow u} f^{r}(x ; u) .
$$

Hence (7Lip) holds.

Besides the examples given in Proposition 7 more elaborated classes of functions have been considered to deal with the subdifferential determination property. Classes of functions and results based on measure and integration theories (e.g. Borwein-Moors [2] or ThibaultZagrodny [22]) are discussed elsewhere. Here, we discuss further the class of functions introduced by L. Thibault and D. Zagrodny in [21]: given a subdifferential $\partial$, a lsc function $g: X \rightarrow]-\infty,+\infty]$ is called $\partial$-subdifferentially and directionally stable ( $s d s$ for short) on $\Omega$ provided that for every $u \in \Omega \cap \operatorname{dom} \partial g$ and $v \in \Omega \cap \operatorname{dom} g$ with $v \neq u$, the following properties hold:

(i) the function $t \mapsto \gamma(t):=g(u+t(v-u))$ is finite and continuous on [0,1];

(ii) for any $t \in\left[0,1\left[\right.\right.$, the right derivative $\gamma_{+}^{\prime}(t)$ exists and is less than $+\infty$; 
(iii) for each fixed $y \in\left[u, v\left[\right.\right.$ and for each real number $\varepsilon>0$, there exists some $\left.r_{0} \in\right] 0,1[$ such that for any $w=y+r(v-u)$ with $\left.r \in] 0, r_{0}\right]$ and for every $\left(x_{n}, x_{n}^{*}\right) \in \partial g$ with $x_{n} \rightarrow x_{0} \in[y, w[$ one has

$$
\limsup _{n \rightarrow \infty}\left\langle x_{n}^{*}, w-x_{n}\right\rangle \leq g^{r}\left(y ; w-x_{0}\right)+\varepsilon\left\|w-x_{0}\right\| .
$$

Proposition 8 (sds implies strictly upper semi-smooth). Let $\Omega \subset X$ a nonempty open convex subset of a Banach space $X$ and let $f: X \rightarrow]-\infty,+\infty]$ be a lsc function with $\Omega \cap \operatorname{dom} f \neq \emptyset$. If the function $f: X \rightarrow]-\infty,+\infty]$ is sds on $\Omega$, then for every $\bar{x} \in \Omega \cap \operatorname{dom} \partial f$, every $u \in X$ with $\bar{x}+u \in \Omega \cap \operatorname{dom} f$ and every $t \in[0,1[$, the function $f$ is strictly upper semismooth at $\bar{x}+t u$ in the direction $u$ and its radial subderivative $f^{r}(\bar{x}+t u ; u)$ is finite.

Proof. Let $f: X \rightarrow]-\infty,+\infty]$ be sds on $\Omega$. Let $\bar{x} \in \Omega \cap \operatorname{dom} \partial f, u \in X$ with $u \neq 0$, such that $\bar{x}+u \in \Omega \cap \operatorname{dom} f$. We apply the above definition of a sds function with $u$ and $v$ respectively replaced by $\bar{x}$ and $\bar{x}+u$. Let $\varepsilon>0$ and let $\bar{x}_{t}:=\bar{x}+t u \in[\bar{x}, \bar{x}+u[$. By condition (iii) of the definition, there exists $\left.r_{0} \in\right] 0,1\left[\right.$ such that for any $w=\bar{x}_{t}+r u$ with $\left.r \in\right] 0, r_{0}$ ] and for every $\left(x_{n}, x_{n}^{*}\right) \in \partial f$ with $x_{n} \rightarrow x_{0} \in\left[\bar{x}_{t}, w[\right.$ one has

$$
\limsup _{n \rightarrow \infty}\left\langle x_{n}^{*}, w-x_{n}\right\rangle \leq f^{r}\left(\bar{x}_{t} ; w-x_{0}\right)+\varepsilon\left\|w-x_{0}\right\| .
$$

Since subdifferentials are densely defined (Theorem 44), for every $x_{0} \in\left[\bar{x}_{t}, w[\right.$ there does exist a sequence $\left(x_{n}, x_{n}^{*}\right) \in \partial f$ with $x_{n} \rightarrow x_{0}$. Given $x_{0} \in\left[\bar{x}_{t}, w\left[\right.\right.$, write $w$ as $w=x_{0}+r_{1} u$ with $r_{1}>0$ so that the above relation can be written as

$$
\limsup _{n \rightarrow \infty}\left\langle x_{n}^{*}, r_{1} u+x_{0}-x_{n}\right\rangle \leq f^{r}\left(\bar{x}_{t} ; r_{1} u\right)+\varepsilon\left\|r_{1} u\right\| .
$$

Dividing by $r_{1}$ we get that for every $x_{0} \in\left[\bar{x}_{t}, w\left[\right.\right.$ and every $\left(x_{n}, x_{n}^{*}\right) \in \partial f$ with $x_{n} \rightarrow x_{0}$,

$$
\limsup _{n \rightarrow \infty}\left\langle x_{n}^{*}, u+\frac{x_{0}-x_{n}}{r_{1}}\right\rangle \leq f^{r}\left(\bar{x}_{t} ; u\right)+\varepsilon\|u\|,
$$

hence, for every $x_{0} \in\left[\bar{x}_{t}, w[\right.$,

$$
\inf _{\alpha \geq 0} \limsup _{x \rightarrow x_{0}} f^{\partial}\left(x ; u+\alpha\left(x_{0}-x\right)\right\rangle \leq f^{r}\left(\bar{x}_{t} ; u\right)+\varepsilon\|u\| .
$$

Invoking Theorem 5, we conclude that for every $x_{0} \in\left[\bar{x}_{t}, w[\right.$, it holds

$$
f^{r}\left(x_{0} ; u\right) \leq \inf _{\alpha \geq 0} \limsup _{x \rightarrow x_{0}} f^{r}\left(x ; u+\alpha\left(x_{0}-x\right)\right\rangle \leq f^{r}\left(\bar{x}_{t} ; u\right)+\varepsilon\|u\| .
$$

The mean value inequality (Proposition 10) produces a point $x_{0} \in\left[\bar{x}_{t}, w\left[\operatorname{such}\right.\right.$ that $f^{r}\left(x_{0} ; u\right)>$ $-\infty$, hence also $f^{r}\left(\bar{x}_{t} ; u\right)>-\infty$ in view of (13). Combining this with condition (ii) of the definition of sds, we see that $f^{r}\left(\bar{x}_{t} ; u\right)$ is finite. Next, considering (13) with $x_{0}=\bar{x}_{t}$ and noting that $\varepsilon>0$ was arbitrary, we see that (8) holds with $\bar{x}=\bar{x}_{t}$, that is, $f$ is strictly upper semismooth at $\bar{x}_{t}$ in the direction $u$.

The next theorem states a key property of (strictly) upper semismooth functions: roughly, a lsc function is (strictly) upper semismooth at some point if and only if its radial subderivative at this point can be recovered from the values of the subdifferential of the function at directional limiting points (at limiting points). 
Theorem 9 (Recovering the radial subderivative from a subdifferential). Let $X$ be a Banach space, $f: X \rightarrow]-\infty,+\infty]$ be lsc, $\bar{x} \in \operatorname{dom} f$ and $u \in X, u \neq 0$. Let also $\partial$ be an arbitrary subdifferential.

(a) Assume $f$ is radially accessible at $\bar{x}$ from $u$. Then $f$ is upper semismooth at $\bar{x}$ in the direction $u$ if and only if

$$
f^{r}(\bar{x} ; u)=\inf _{\alpha \geq 0} \limsup _{x \rightarrow u \bar{x}} f^{\partial}(x: u+\alpha(\bar{x}-x)) .
$$

(b) $f$ is strictly upper semismooth at $\bar{x}$ in the direction $u$ if and only if

$$
f^{r}(\bar{x} ; u)=\inf _{\alpha \geq 0} \limsup _{x \rightarrow \bar{x}} f^{\partial}(x: u+\alpha(\bar{x}-x)) .
$$

Proof. (a) By Theorem 5 with $v=u$, the formulas (14) and (7) bis) are the same.

(b) We observed that equality holds in (8); this equality and (15) are the same according to Theorem 5 with $v=0$.

\section{Subdifferential determination property}

The two theorems of this section assert, with slightly different assumptions, that the upper semismooth functions have the subdifferential determination property.

Theorem 10 (Subdifferential determination property). Let $X$ be a Banach space and $\Omega \subset X$ be a nonempty open convex subset. Let $f, g: X \rightarrow]-\infty,+\infty]$ be lsc with $\Omega \cap \operatorname{dom} f \neq \emptyset$. Assume that for every $\bar{x} \in \Omega \cap \operatorname{dom} \partial f \cap \operatorname{dom} \partial g$ and every $u \in X, u \neq 0$, with $\bar{x}+u \in$ $\Omega \cap \operatorname{dom} f \cap \operatorname{dom} g$, the points $\bar{x}_{t}:=\bar{x}+t u$ satisfy the following properties:

(10,1) $t \mapsto f\left(\bar{x}_{t}\right)$ and $t \mapsto g\left(\bar{x}_{t}\right)$ are continuous on $[0,1]$;

(10,2) there is a countable subset $C \subset[0,1]$ such that for every $t \in[0,1] \backslash C$, either $f_{+}^{r}\left(\bar{x}_{t} ; u\right)$ or $g^{r}\left(\bar{x}_{t} ; u\right)$ is finite, and $g$ is upper semismooth at $\bar{x}_{t}$ in the direction $u$.

Then,

$$
\partial f(x) \subset \partial g(x) \text { for all } x \in \Omega \cap \operatorname{dom} f \Longrightarrow f=g+\text { const on } \Omega \cap \operatorname{dom} f .
$$

Proof. Assume

$$
\partial f(x) \subset \partial g(x) \text { for all } x \in \Omega \cap \operatorname{dom} \partial f .
$$

The beginning of the proof is the same as in Thibault-Zagrodny [22, Theorem 3.21]. We may suppose that $\Omega \cap \operatorname{dom} f$ is not a singleton because otherwise the result is obvious. Then, if $\Omega \cap \operatorname{dom} f$ contains two distinct points $x, y$, the set $\Omega \cap \operatorname{dom} \partial f$ also contains two distinct points $\bar{x}, \bar{y}$ by the density of dom $\partial f$ into $\operatorname{dom} f$ (Theorem 44). From (17) it follows that $\bar{x}$ and $\bar{y}$ also belong to $\operatorname{dom} \partial g \subset \operatorname{dom} g$.

First step. Fix $\bar{x} \in \Omega \cap \operatorname{dom} \partial f=\Omega \cap \operatorname{dom} \partial f \cap \operatorname{dom} \partial g$ and $\bar{y} \in \Omega \cap \operatorname{dom} f \cap \operatorname{dom} g$, with $\bar{x} \neq \bar{y}$. We claim that

$$
f(\bar{y})-f(\bar{x}) \leq g(\bar{y})-g(\bar{x}) .
$$

Let $u:=\bar{y}-\bar{x}$, hence $\bar{x}_{t}=\bar{x}+t u=\bar{x}+t(\bar{y}-\bar{x})$, and let $t \in[0,1] \backslash C$. By Assumption (10,1), $f$ is radially accessible at $\bar{x}_{t}$ from $u$, so in view of Proposition 6 and Theorem 5

$$
f_{+}^{r}\left(\bar{x}_{t} ; u\right) \leq \inf _{\alpha \geq 0} \limsup _{x \rightarrow{ }_{u} \bar{x}_{t}} f^{\partial}\left(x ; u+\alpha\left(\bar{x}_{t}-x\right)\right) .
$$


By Assumption (10,2), $g$ is upper semismooth at $\bar{x}_{t}$ in the direction $u$, hence according to Definition 1 and Theorem 5

$$
\inf _{\alpha \geq 0} \limsup _{x \rightarrow u} g_{\bar{x}_{t}} g^{\partial}\left(x ; u+\alpha\left(\bar{x}_{t}-x\right)\right) \leq g^{r}\left(\bar{x}_{t} ; u\right) .
$$

From (17), the right-hand side of (19) is less than or equal to the left-hand side of (20). We therefore conclude that

$$
f_{+}^{r}\left(\bar{x}_{t} ; u\right) \leq g^{r}\left(\bar{x}_{t} ; u\right) \text { for all } t \in[0,1] \backslash C .
$$

Now, consider the functions $\varphi: t \in[0,1] \mapsto f\left(\bar{x}_{t}\right)$ and $\gamma: t \in[0,1] \mapsto g\left(\bar{x}_{t}\right)$. By Assumption (10,1), they are finite and continuous on [0,1] and, by Assumption (10,2), for every $t \in[0,1] \backslash C$, either $\varphi_{+}^{r}(t ;+1)$ or $\gamma^{r}(t ;+1)$ is finite. On the other hand, (21) can be reformulated as

$$
\varphi_{+}^{r}(t ;+1) \leq \gamma^{r}(t ;+1) \text { for all } t \in[0,1] \backslash C .
$$

So we may invoke Proposition 3 to derive that $\varphi(1)-\varphi(0) \leq \gamma(1)-\gamma(0)$, that is, (18) holds.

Second step. In the first step, we have shown that (18) holds for every point $\bar{x} \in \Omega \cap \operatorname{dom} \partial f$ and $\bar{y} \in \Omega \cap \operatorname{dom} f \cap \operatorname{dom} g$. Now, let $\bar{x} \in \Omega \cap \operatorname{dom} f$ and $\bar{y} \in \Omega \cap \operatorname{dom} f \cap \operatorname{dom} g$. Applying the density Theorem 4, we find a sequence $\left(\bar{x}_{n}\right)_{n}$ in dom $\partial f$ such that $\bar{x}_{n} \rightarrow \bar{x}$ and $f\left(\bar{x}_{n}\right) \rightarrow f(\bar{x})$. By (18), for every $n \in \mathbb{N}$ and $\bar{y} \in \Omega \cap \operatorname{dom} f \cap \operatorname{dom} g$ it holds

$$
f(\bar{y})-f\left(\bar{x}_{n}\right) \leq g(\bar{y})-g\left(\bar{x}_{n}\right) .
$$

Because $f\left(\bar{x}_{n}\right) \rightarrow f(\bar{x})$ and $g$ is lower semicontinuous at $\bar{x}$, passing to the limit we get

$$
f(\bar{y})-f(\bar{x}) \leq g(\bar{y})-g(\bar{x}) .
$$

This inequality shows that $g(\bar{x})$ is finite whenever $\bar{x} \in \Omega \cap \operatorname{dom} f$, that is, $\Omega \cap \operatorname{dom} f=$ $\Omega \cap \operatorname{dom} f \cap \operatorname{dom} g$. So finally one has

$$
f(\bar{y})-f(\bar{x}) \leq g(\bar{y})-g(\bar{x}) \text { for all } \bar{x}, \bar{y} \in \Omega \cap \operatorname{dom} f .
$$

Then, interchanging the role of $\bar{x}$ and $\bar{y}$, we derive that in fact

$$
f(\bar{y})-f(\bar{x})=g(\bar{y})-g(\bar{x}) \text { for all } \bar{x}, \bar{y} \in \Omega \cap \operatorname{dom} f,
$$

which means that $f=g+$ const on $\Omega \cap \operatorname{dom} f$. The proof is complete.

If instead of the continuous version of the mean value theorem (Proposition 3) we use the semicontinuous version (Proposition 2), we obtain a variant of the above theorem with weaker assumptions on $f$ but stronger on $g$. The proof being similar will not be repeated.

Theorem 11 (Subdifferential determination property: semicontinuous variant). Let $X$ be a Banach space and $\Omega \subset X$ be a nonempty open convex subset. Let $f, g: X \rightarrow]-\infty,+\infty]$ be lsc with $\Omega \cap \operatorname{dom} f \neq \emptyset$. Assume that for every $\bar{x} \in \Omega \cap \operatorname{dom} \partial f \cap \operatorname{dom} \partial g$ and every $u \in X$, $u \neq 0$, with $\bar{x}+u \in \Omega \cap \operatorname{dom} f \cap \operatorname{dom} g$, the points $\bar{x}_{t}:=\bar{x}+$ tu satisfy the following properties:

(11,1) $t \mapsto g\left(\bar{x}_{t}\right)$ is continuous on $[0,1]$; 
(11,2) for every $t \in\left[0,1\left[\right.\right.$, either $f^{r}\left(\bar{x}_{t} ; u\right)$ or $g^{r}\left(\bar{x}_{t} ; u\right)$ is finite, and either (a) or (b) holds:

(a) $f$ is radially accessible at $\bar{x}_{t}$ from $u$ and $g$ is upper semismooth at $\bar{x}_{t}$ in direction $u$,

(b) $g$ is strictly upper semismooth at $\bar{x}_{t}$ in direction $u$.

Then,

$$
\partial f(x) \subset \partial g(x) \text { for all } x \in \Omega \cap \operatorname{dom} f \Longrightarrow f=g+\text { const on } \Omega \cap \operatorname{dom} f .
$$

Remark 11.1. (a) Theorem [10 is new in the context of mean-valued based theorems. Its assumption on $g$ is much weaker than the one in Theorem 11] It should rather be compared and contrasted with integration-based results such as those in [2, 22. Since the technique and concepts are totally different, this will be done in a separate paper.

(b) Theorem 11 unifies several results. As an illustration, we mention three of them, which cannot be derived from each other but which are all special cases of Theorem 11, since the functions they involve are either upper semismooth (case b1) or strictly upper semismooth (cases b2 and b3).

(b1) Correa-Jofré [5, Proposition 5.4], where $g$ is locally Lipschitz, semismooth and whose Clarke subdifferential is single-valued at any point of a dense subset of $X$;

(b2) Thibault-Zlateva [23, Theorem 3.3], where lsc regular functions $g$ which are continuous relative to their domains and strictly directionally Lipschitz are shown to have a "local subdifferential determination property";

(b3) Thibault-Zagrodny [21, Theorem 4.1] (in the special case $\gamma=0$ ), where $g$ is sds.

\section{References}

[1] J. M. Borwein. Generalisations, examples and counter-examples in analysis and optimisation. Set-valued Var. Anal., in press.

[2] J. M. Borwein and W. B. Moors. A chain rule for essentially smooth Lipschitz functions. SIAM J. Optim., 8(2):300-308, 1998.

[3] J. M. Borwein, W. B. Moors, and X. Wang. Generalized subdifferentials: a Baire categorical approach. Trans. Amer. Math. Soc., 353(10):3875-3893, 2001.

[4] J. M. Borwein and H. M. Strójwas. The hypertangent cone. Nonlinear Anal., 13(2):125-144, 1989.

[5] R. Correa and A. Jofré. Tangentially continuous directional derivatives in nonsmooth analysis. J. Optim. Theory Appl., 61(1):1-21, 1989.

[6] A. Daniilidis and P. Georgiev. Approximate convexity and submonotonicity. J. Math. Anal. Appl., 291(1):292-301, 2004.

[7] A. Daniilidis, F. Jules, and M. Lassonde. Subdifferential characterization of approximate convexity: the lower semicontinuous case. Math. Program., 116(1-2, Ser. B):115-127, 2009.

[8] J. Dieudonné. Foundations of modern analysis. Academic Press, New York-London, 1969. Enlarged and corrected printing, Pure and Applied Mathematics, Vol. 10-I.

[9] A. D. Ioffe. On the theory of subdifferentials. Adv. Nonlinear Anal., 1(1):47-120, 2012.

[10] F. Jules and M. Lassonde. Subdifferential estimate of the directional derivative, optimality criterion and separation principles. Optimization, 62(9):1267-1288, 2013. 
[11] F. Jules and M. Lassonde. Subdifferential test for optimality. J. Global Optim., 59(1):101-106, 2014.

[12] M. Lassonde. Subderivative-subdifferential duality formula. arXiv:1611.04045v2

[13] R. Mifflin. Semismooth and semiconvex functions in constrained optimization. SIAM J. Control Optimization, 15(6):959-972, 1977.

[14] J.-J. Moreau. Proximité et dualité dans un espace hilbertien. Bull. Soc. Math. France, 93:273-299, 1965.

[15] J.-P. Penot. Calculus without derivatives, volume 266 of Graduate Texts in Mathematics. Springer, New York, 2013.

[16] R. T. Rockafellar. On the maximality of sums of nonlinear monotone operators. Trans. Amer. Math. Soc., 149:75-88, 1970.

[17] R. T. Rockafellar. Directionally Lipschitzian functions and subdifferential calculus. Proc. London Math. Soc. (3), 39(2):331-355, 1979.

[18] R. T. Rockafellar. Generalized directional derivatives and subgradients of nonconvex functions. Canad. J. Math., 32(2):257-280, 1980.

[19] R. T. Rockafellar. Favorable classes of Lipschitz-continuous functions in subgradient optimization. In Progress in nondifferentiable optimization, volume 8 of IIASA Collaborative Proc. Ser. CP-82, pages 125-143. Internat. Inst. Appl. Systems Anal., Laxenburg, 1982.

[20] J. E. Spingarn. Submonotone subdifferentials of Lipschitz functions. Trans. Amer. Math. Soc., 264(1):77-89, 1981.

[21] L. Thibault and D. Zagrodny. Enlarged inclusion of subdifferentials. Canad. Math. Bull., 48(2):283-301, 2005.

[22] L. Thibault and D. Zagrodny. Subdifferential determination of essentially directionally smooth functions in Banach space. SIAM J. Optim., 20(5):2300-2326, 2010.

[23] L. Thibault and N. Zlateva. Integrability of subdifferentials of directionally Lipschitz functions. Proc. Amer. Math. Soc., 133(10):2939-2948 (electronic), 2005. 